\title{
Network Structure in a Link-formation Game: An Experimental Study
}

by

\author{
Alexander Elbittar, Rodrigo Harrison and Roberto Muñoz *
}

\begin{abstract}
Network formation is frequently modeled using link-formation games and typically present a multiplicity of Nash equilibria. Cooperative refinements - such as strong or coalitional proof Nash equilibria - have been the standard tool used for equilibrium selection in these games. Non-cooperative refinements derived from the theory of global games have shown also that, for a class of payoff functions, multiplicity of equilibria disappears when the game is perturbed by introducing small amounts of incomplete information. We conducted a laboratory study evaluating the predictive power of each of these refinements in an illustrative link-formation game. Compared with cooperative game solutions, the global game approach did significantly better at predicting the strategies played by individuals in the experiment.

Keywords: Networks, Global Games, Cooperative Games, Equilibrium Selection, Experimental economics.
\end{abstract}

JEL Classification Numbers: C70, C92, D20, D44, D82.

\footnotetext{
${ }^{*}$ We would like to thank Carlos Lever for helpful suggestions and for collaboration in the experimental sessions, Alvaro Parra and Rodrigo Saucedo for research assistance, and Abelardo León for assistance in programming. We also thank Cesar Martinelli, Nicolas Melissas and Ricardo Smith for useful conversations. We are also grateful to seminar participants at CIDE, ITAM, George Mason University and PUC, the 2007 ESA World Meetings in Rome, the 2007 SECHI Meetings in Chile, and the 2007 Workshop on Global Games in Stony Brook. Financial support from CIDE and Pontificia Universidad Católica de Chile is acknowledge. To contact Elbittar: División de Economía, CIDE. E-mail: alexander.elbittar@cide.edu; Harrison: Instituto de Economía Pontificia Universidad Católica de Chile. E-mail: harrison@faceapuc.cl; Muñoz: División de Economía, Departamento de Industrias, Universidad Técnica Federico Santa María. E-mail: roberto.munoz@usm.cl.
} 


\section{Introduction}

Social and economic interactions have been modeled using network structures or graphs, where agents are represented by nodes and their relationships are represented by arcs between the nodes. These network structures play an important role in many forms of economic situations and have been studied widely. For instance, Montgomery (1991) showed how patters of social ties between individuals play a critical role in labor market outcomes. Fafchamps and Lund (2003) showed how mutual insurance in rural areas takes place within networks.

Myerson (1991) proposed a class of link-formation games for modeling network formation. In this class of games, agents simultaneously choose which agents they want to be connected to and a link is formed whenever they both agree. This class of games proved to be successful in the study of many specific theoretical problems (Dutta and Jackson, 2001; Jackson, 2003).

However, it is also commonly known that link-formation games can lead to multiple network configurations supported by multiple Nash equilibria (Jackson, 2003). Under some circumstances, any network within this class of games can be supported by a multiplicity of Nash equilibria (Slikker and van den Nouweland, 2000).

Jackson (2003) argued that in order to deal with the fact that it takes two players to form a link, we needed something beyond the traditional non-cooperative refinements, such as strong Nash equilibrium $(S N E)$, coalition proof Nash equilibrium $(C P N E)$, or pairwise strong Nash equilibrium (PSNE) (Dutta and Mutuswami, 1997 and Jackson, 2004).

On the other hand, Bala and Goyal (2000) and Harrison and Muñoz (2007) argued that, in the same network context, non-cooperative equilibrium selection approaches are still feasible and meaningful. Using the theory of global games, Harrison and Muñoz (2007) showed that the multiplicity of Nash equilibria arising in a class of link-formation games, disappears when the game is perturbed by introducing small amounts of incomplete information into the payoff functions.

The experiment reported here compares the accuracy of the theory of global game and the alternative cooperative refinements, such as $S N E$, predicting the network configuration in a link-formation game. In particular, we consider an illustrative link-formation game where agents obtain benefits and bear costs for establishing valuable links and where the network configurations predicted by these refinements are radically different. In order to check for robustness, we review different comparative static predictions for various parameters of the payoff function and different degrees of players' information.

This paper is related to previous experimental studies on network formation (see Kosfeld, 2004). In contrast to our paper, most of the research on this topic has concentrated on testing the predictive power of cooperative refinements, such as PSNE, (Johnson and 
Gilles, 2004) and non-cooperative refinements, such as strict Nash equilibrium, (Callander and Plott, 2005 and Falk and Kosfeld, 2005) without a cross comparison.

There are other experimental studies that tested the accuracy of global games predictions (Heinemann et al., 2004; Cabrales et al., 2007; and Shurchkov, 2007). We depart from them in two important aspects. First, as far as we know, this is the first application to study network formation. Second, previous studies have shown that the global games approach is very useful identifying the unique equilibrium under incomplete information, but it does not work as well as a refinement of the related complete information game.

The rest of this paper is organized as follows: Section 2 develops the connection model and derives testable predictions. Section 3 discusses experimental design. Section 4 presents laboratory results. Section 5 concludes.

\section{The Connection Model}

Harrison and Muñoz (2007) consider a simple static link-formation game of complete information $G_{x}$. This game is played by three players in the set $N=\{1,2,3\}$, indexed by $i$. Each player has a set of strategies $A_{i}=\{0,1\}^{2}$. The strategy for player $i$ is a vector which identifies the set of players she wants to be connected to; for example, the strategy $a_{i}=\left(a_{i j}=1, a_{i k}=0\right)$ represents player $i$ 's intention to connect with player $j$ but not with player $k$. Players simultaneously choose strategies. Next, a link between two of them will be formed if and only if both players want to form the link. The resulting network $g$ is a list of the pairs of players that are linked to each other. Formally, $g(a)=\{i j: i, j \in N$ such that $\left.a_{i j}=a_{j i}=1\right\}$.

The payoff structure considered is simple and can be viewed as a variation of the Jackson and Wolinsky (1996) connection model where links can be interpreted as friendship relations. ${ }^{1}$

$$
\pi_{i}\left(a_{i}, a_{-i}, x\right)=(\alpha x-c) a_{i j}+a_{i j} a_{j i}\left(x+a_{j k} a_{k j} \beta x\right)+(\alpha x-c) a_{i k}+a_{i k} a_{k i}\left(x+a_{k j} a_{j k} \beta x\right)
$$

The variable $x$ defines the level of benefit that agent $i$ obtains for each link she wants to form. Parameter $c$ is the fixed cost that agent $i$ incurs for each link she wants to form. We then assume that parameters $\alpha$ and $\beta$ belong to the interval $(0,1)$ since linking benefits are scaled down in relation to those obtained from direct reciprocity.

Benefits for player $i$ in equation (1) come from three components: independent, direct and indirect connections to other individuals. The independent component is what player $i$ receives, $(\alpha x-c)$, by unilaterally requesting a link to another player, even if the link is not

\footnotetext{
${ }^{1}$ Alternatively, for agent $i$ agent $j$ does not have an intrinsic value. Instead, what increases agent $i$ 's payoff is the link with agent $j$. In other words, agent $i$ does not receive benefits from $j$, but from her friendship with $j$.
} 
finally formed. If player $i$ requests a link to player $j$, and player $j$ shows the corresponding link intention to player $i$, then $i$ 's benefit increases in $x$ as a result of a direct link created between $i$ and $j$. Finally, there also exists benefits for player $i$ arising from the indirect connections with the other agents. For example, if player $j$ has formed a direct link with player $k$, then player $i$ gets the indirect benefit of $\beta x$.

The connection model with the above payoff structure presents a multiplicity of Nash equilibria. Figure 1 provides a summary of the different network structures supported by a strategy profile in the set of Nash equilibria, $N E\left(G_{x}\right)$, for different values of $x$. Several cases are considered:

Case (i): If $x<\underline{x}=c /(1+\alpha+\beta)$, then the dominant strategy for any player $i$ is to play $a_{i}=(0,0) \equiv \mathbf{0}$ and the empty network emerges.

Case (ii): If $c /(1+\alpha+\beta)<x<c /(1+\alpha)$, it is easy to see that the best response correspondence of player $i$ depends on the existence of the link between players $j$ and $k$.

$$
B R_{i}\left(a_{-i}\right)= \begin{cases}a_{i j}=1, a_{i k}=1 & \text { if } \quad a_{j i}=a_{k i}=a_{j k}=a_{k j}=1 \\ a_{i j}=1, a_{i k}=0 & \text { if } \quad a_{j i}=a_{j k}=a_{k j}=1, a_{k i}=0 \\ a_{i j}=0, a_{i k}=1 & \text { if } \quad a_{j i}=0, a_{k i}=a_{j k}=a_{k j}=1 \\ a_{i j}=0, a_{i k}=0 & \text { otherwise }\end{cases}
$$

It is possible to prove that in this case, only the empty and the complete network can be supported by strategy profiles in $N E\left(G_{x}\right)$.

Case (iii): If $c /(1+\alpha)<x<c / \alpha$, the strategies of agents $j$ and $k$ in relation to their connection do not affect the best response correspondence of agent $i$.

$$
B R_{i}\left(a_{-i}\right)= \begin{cases}a_{i j}=1, a_{i k}=1 & \text { if } \quad a_{j i}=a_{k i}=1 \\ a_{i j}=1, a_{i k}=0 & \text { if } \quad a_{j i}=1, a_{k i}=0 \\ a_{i j}=0, a_{i k}=1 & \text { if } \quad a_{j i}=0, a_{k i}=1 \\ a_{i j}=0, a_{i k}=0 & \text { if } \quad a_{j i}=0, a_{k i}=0\end{cases}
$$

In this case, direct connections are enough to guarantee profitability. This characteristic leads to a multiplicity of Nash equilibria. Even more, it is possible to prove that every feasible network among the three agents can be supported by a profile in $N E\left(G_{x}\right)$.

Case (iv): If $x>\bar{x}=c / \alpha$, then the dominant strategy is to form links with all the other players $a_{i}=(1,1) \equiv \mathbf{1}$, resulting in a complete network.

As is shown in Figure 1, since multiplicity arises in this link-formation game, we cannot predict exactly which network structure will emerge in equilibrium. 


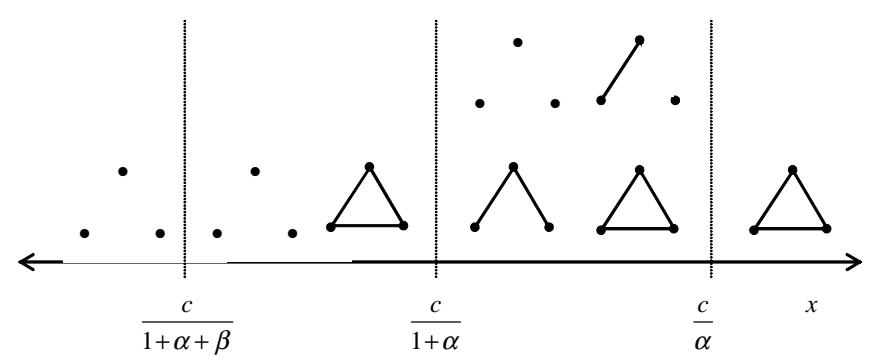

Figure 1: Network structures supported by Nash Equilibria in the Connection Model

\subsection{Equilibrium Selection Using Cooperative Refinements}

In general, the network literature (see Dutta et al., 1998) has used cooperative approaches, such as $S N E$ or $C P N E$, to solve this equilibrium selection problem. This approach is close to the idea of strong stability. This means that its application relies on the players' ability to deviate (not necessarily unilaterally) from some profile, candidate to be an equilibrium. $^{2}$

A strategy profile is called a $S N E$ if it is a Nash equilibrium and there is no coalition of players that can strictly increase the payoffs of all its members by jointly deviating (Aumann, 1959; Dutta and Mutuswami, 1997). Another commonly used coalitional refinement is $C P N E$. A strategy profile is a $C P N E$ if no coalition can deviate to a profile that strictly improves the payoffs of all the players in the coalition. However, the set of admissible deviations is smaller, because they have to be stable with respect to further deviations by sub-coalitions (Dutta and Mutuswami, 1997). ${ }^{3}$

In our three player game $G_{x}$, the set of $S N E\left(G_{x}\right)$ coincides with the set of $C P N E\left(G_{x}\right)$. The application of these coalitional refinements is very direct and the analysis must be performed in separated areas. It is easy to see that the strategy profile $a=(\mathbf{0}, \mathbf{0}, \mathbf{0}) \equiv[\mathbf{0}]$ is the unique profile in $\operatorname{SNE}\left(G_{x}\right)$ when $x<c /(1+\alpha+\beta)$, because for this range of values, each agent plays $\mathbf{0}$ as a dominant strategy and, consequently, no coalition of agents can improve upon it. ${ }^{4}$

On the other hand, $a=(\mathbf{1}, \mathbf{1}, \mathbf{1}) \equiv[\mathbf{1}]$ is the unique profile in $\operatorname{SNE}\left(G_{x}\right)$ when $x>$ $c /(1+\alpha+\beta)$. In order to understand this, consider any other given strategy profile $\widehat{a} \neq[\mathbf{1}]$ (Nash equilibrium or not). Because of the complementarities involved in the

\footnotetext{
${ }^{2}$ Other cooperative concepts, such as PSNE, can also be applied. However, their predictions are not unique for this particular game (Harrison and Muñoz, 2007).

${ }^{3}$ The relations between the sets of strategy profiles for a general game $G$ is the following:

$\operatorname{SNE}(G) \subseteq \operatorname{CPNE}(G)$

${ }^{4}$ In what follows $[\mathbf{0}]$ and $[\mathbf{1}]$ represents a matrix full of zeros or ones respectively. The dimensionality is given by the profile they are representing. For example, in this three players case, $a=[\mathbf{0}]$ is a $2 \times 3$ matrix of zeros representing a complete strategy profile and $a_{-i}=[\mathbf{0}]$ is a $2 \times 2$ matrix of zeros representing a strategy profile that excludes player $i$ 's strategy.
} 
payoff function (1), the grand coalition, by playing $a=[\mathbf{1}]$, can strictly increase the payoffs of all its members. In this case, $\widehat{a}$ is not in $S N E\left(G_{x}\right)$. Additionally, starting from $a=[\mathbf{1}]$ which is trivially a Nash equilibrium, it is not possible to increase the payoff of any player in a deviation. Finally, if $x=\underline{x}=c /(1+\alpha+\beta)$, then $\operatorname{SNE}\left(G_{x}\right)=\{[\mathbf{0}],[\mathbf{1}]\}$.

\subsection{Equilibrium Selection Using the Global Games Approach}

Harrison and Muñoz (2007) were able to select a unique equilibrium profile by using a non-cooperative refinement, derived from the global games literature (see Morris and Shin, 2002). The idea is to perturb the payoff function by introducing an arbitrarily small amount of incomplete information. In this way, it is possible to find the equilibrium in the perturbed game and establish implications for the complete information game. In the following we formally describe the procedure and establish the result that we are interested in testing.

Suppose that $G_{x}$ is a game of incomplete information. The amount of incomplete information is parameterized by $\sigma$, so we call this game $G(\sigma)$ and we define $B N E(G(\sigma))$ as the set of Bayesian Nash equilibria of $G(\sigma)$. Player $i$ 's payoff function is still given by equation (1), but now it depends on the private value $x_{i}$, such that each player's value has the following structure: $x_{i}=x+\sigma \varepsilon_{i}$, where $\sigma>0$ is a scale factor, $x$ is drawn from the interval $[\underline{X}, \bar{X}]$ with a uniform density, and $\varepsilon_{i}$ is a random variable distributed according to a continuous density $\varphi$ with support in the interval $[-1,1]$. We also assume that $\varepsilon_{i}$ is iid across the individuals. Now, players valuations are related, so that $x_{i}$ also constitutes a noisy signal of $x_{j}$ for all $j \neq i: x_{j}=x_{i}+\sigma\left(\varepsilon_{j}-\varepsilon_{i}\right)$.

This noise structure has been used in the global games literature for modeling the conditional distribution of the opponents' values $x_{-i}$, given player $i$ 's own value, $x_{i}$. The conditional distribution admits a continuous density $f_{\sigma}$ and a $c d f F_{\sigma}$ with support in $V \equiv\left[x_{i}-2 \sigma, x_{i}+2 \sigma\right]^{2}$.

Under incomplete information, the pure strategy for player $i$ is the function $s_{i}:[\underline{X}-$ $\sigma, \bar{X}+\sigma] \rightarrow A_{i}$. The set of all these functions is denoted $S_{i}$. We also define $S_{-i}=\times_{j \neq i} S_{j}$. Thus, a complete strategy profile $s=\left(s_{i}, s_{-i}\right) \in S \equiv S_{i} \times S_{-i}$. Therefore, the switching strategy between the lowest and the highest action with threshold $k_{i}$ is the pure strategy satisfying:

$$
s_{i}^{k_{i}}\left(x_{i}\right)=\left\{\begin{array}{ccc}
\mathbf{1} & \text { if } & x_{i}>k_{i} \\
\mathbf{0} & \text { if } & x_{i}<k_{i}
\end{array}\right.
$$

The application of this equilibrium selection approach to our problem is described in the following proposition. 


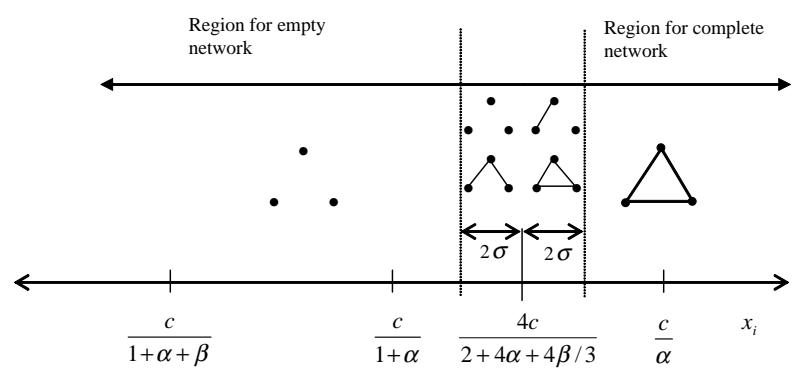

Figure 2: Networks that player $i$ predicts, given $x_{i}$.

Proposition 1 (Harrison and Muñoz, 2007) Consider the game $G(\sigma)$. For any $\sigma>$ $0, s_{i}^{k^{*}}\left(x_{i}\right)$ is the only strategy profile that survives the iterated elimination of strictly dominated strategies in $G(\sigma)$, where:

$$
s_{i}^{k^{*}}\left(x_{i}\right)=\left\{\begin{array}{lll}
\mathbf{1} & \text { if } & x_{i}>k^{*} \\
\mathbf{0} & \text { if } & x_{i}<k^{*}
\end{array} \quad \forall i \text { and } k^{*}=\frac{4 c}{2+4 \alpha+4 \beta / 3}\right.
$$

This result shows that the perturbed connection model $G(\sigma)$ has a unique strategy profile $s^{*}$ played in equilibrium, which is independent of $\sigma$. In this profile every player play a switching strategy $s_{i}^{k^{*}}\left(x_{i}\right)$, with $k^{*}=\frac{4 c}{2+4 \alpha+4 \beta / 3}$.

Proposition 1 proves that each player uses switching strategy $s_{i}^{*}$ that is independent of the noise size $\sigma .^{5}$ However, the network formed depends on $\sigma$. In general, if some $x_{i}>k^{*}+$ $2 \sigma$, then every player receives a value greater than $k^{*}$ and therefore the complete network is formed. Equivalently, if some $x_{i}<k^{*}-2 \sigma$, the empty network is formed. Finally, if $x_{i}$ belongs to interval $\left[k^{*}-2 \sigma, k^{*}+2 \sigma\right]$, then any network can be formed depending on the realization of each player's value. Following this analysis it is easy to see that as $\sigma$ goes to zero, just two possibilities remain, the empty and the complete network. The equilibrium profile $s^{*}$ selected in $G(\sigma)$ does not depend on the size of noise $\sigma$.

Figure 2 shows the networks that player $i$ is able to predict based on her private value $x_{i}$. If $x_{i}<k^{*}-2 \sigma$, then player $i$ can be sure that the signals received by other players are also lower than $k^{*}$, and given the equilibrium strategies $s_{i}^{*}\left(x_{i} ; k^{*}\right)$, everybody else will play the action $\mathbf{0}$. An analogous situation occurs when $x_{i}>k^{*}+2 \sigma$. Then, all the players will select action 1. However, if the signal received satisfies $k^{*}-2 \sigma<x_{i}<k^{*}+2 \sigma, x_{j}$ can be higher or lower than $k^{*}$ and then any network can arise. When $\sigma$ goes to zero, i.e. when the amount of incomplete information tends to disappear, the middle area is

\footnotetext{
${ }^{5}$ Note that $s_{i}^{*}$ also is independent of the noise structure $\varphi$. In addition, we have assumed that the parameter $x$ is distributed according to a flat prior, but it is possible to prove that any prior can be treated as a flat prior when $\sigma$ goes to zero.
} 
reduced to a zone of an arbitrarily small width.

\subsection{Efficient Allocation of the Game}

A strategy profile $a_{x}$ is efficient for the game $G_{x}$ if it maximizes the sum of the payoffs of the players. The set of efficient strategy profiles for the game $G_{x}$ is denoted by $E\left(G_{x}\right)$. It is easy to check that in our example the efficient strategy profiles are:

$$
a_{x}= \begin{cases}{[\mathbf{0}]} & \text { if } x<c /(1+\alpha+\beta) \\ {[\mathbf{1}]} & \text { if } x>c /(1+\alpha+\beta)\end{cases}
$$

and as a consequence, the actions played in the equilibrium selected in the global games approach are not efficient. Harrison and Muñoz (2007) also proved that the efficient allocation is a stable allocation under cooperative refinements, such as $S N E$.

A summary of the predicted network configurations is contained in Figure 3. Part (a) shows the networks supported by the Nash equilibria $(N E)$ in the connection model. Part (b) shows the efficient networks, supported by $S N E$. Part (c) contains the networks supported by the $B N E$ according to the global games approach when $\sigma$ goes to zero.

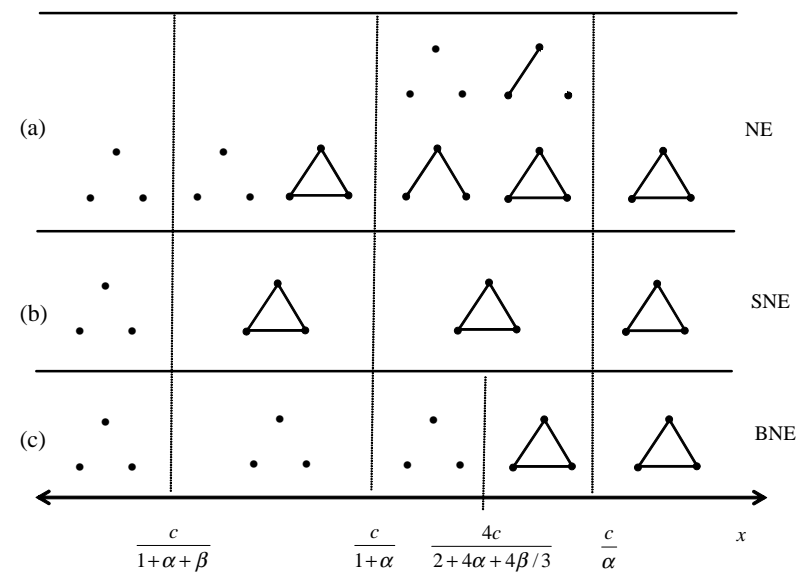

Figure 3: Networks supported by the Nash Equilibria (NE), the Strong Nash Equilibrium, (SNE) and the Bayesian Nash Equilibrium in the Global Games approach (BNE). 
Table 1: Experimental Design

\begin{tabular}{|c|c|c|c|c|c|c|}
\hline Treatment & Alpha & Connection & Perturbation & \multicolumn{2}{|c|}{ Predicted Threshold } & Number of \\
\cline { 5 - 6 } & $(\alpha)$ & Cost $(c)$ & Size $(\sigma)$ & Global Games $\left(k^{*}\right)$ & SNE $(\underline{x})$ & Sessions \\
\hline \hline 1 & 0.6 & 120 & 0 & 103 & 67 & 3 \\
\hline 2 & 0.4 & 120 & 0 & 124 & 75 & 3 \\
\hline 3 & 0.6 & 180 & 0 & 154 & 100 & 3 \\
\hline 4 & 0.4 & 180 & 0 & 186 & 113 & 2 \\
\hline 5 & 0.4 & 120 & 10 & 124 & 75 & 2 \\
\hline 6 & 0.4 & 120 & 50 & 124 & 75 & 1 \\
\hline
\end{tabular}

Note 1: In all these treatments, the parameter $\beta$ was equal to 0.2 .

Note $2: k^{*}=\frac{4 c}{2+4 \alpha+4 \beta / 3}$ and $\underline{x}=\frac{c}{(1+\alpha+\beta)}$

\section{Experimental Designs}

\subsection{Structure of the Network Formation Game}

Our experimental design looks at the outcomes of a link-formation game. In this game, each member of a group of three players decides whether to request a link to each of the other two members of the group after observing her connection value. Our goal is to experimentally check how powerful the global games refinement is with respect to cooperative refinements, like $S N E$. Therefore, we implement our experimental design of this link-formation game under complete information for different parameters values of alpha, $\alpha$, and connection cost, $c$. This design allows us to check whether subjects are able to coordinate around specific switching thresholds for either requesting or not requesting a link, and to see how close the actual switching thresholds to theoretical predictions are.

Table 1 summarizes the parameter values, the expected switching thresholds, and the number of sessions per treatment. The first three treatments check the predicted power of each solution concept. They also check the comparative statics derived from the linkformation game. The fourth treatment allows us to check the robustness of our results when the dominant payoff region is deleted from the upper support of $x$.

In the last three treatments, we look at the impact of different values of the perturbation parameter $\sigma$ on subjects' probability to request links to other members of their group and on the variability of the actual switching thresholds. Thus, these treatments will allow us to check the robustness of the global games approach under an incomplete information setup.

\subsection{Design Parameters}

This section describes the general experimental procedure.

Participants and Venue. Subjects were drawn from a wide cross-section of undergrad- 
uate and graduate students at Pontificia Universidad Católica de Chile (PUC) in Santiago, Chile. Each subject was only permitted to participate in one session. The experiment was run using computers.

Number of Periods. To familiarize subjects with the procedures, ten practice periods were conducted in each session prior to the fifty real (affecting monetary payoff) periods. ${ }^{6}$

Matching Procedure and Group Size. At the beginning of each period, the computer randomly formed groups of three participants, so that each participant formed part of a new group in each of the following periods. Furthermore, participants did not know who they were grouped with in any given period. In each session there were 15 participants.

Link Procedure and Payoff Structure. All participants were informed that they have to decide in every period whether to request a link to zero, one, or two members of the group. They were told that a participant monetary payoff for each link-request would be equal to the sum of the following three components: i) Independent connection component $(=\alpha \times x-c)$ : the payoff a participant would get if she requested a link to another participant. ii) Direct (or complete) connection component $(=x)$ : the payoff a participant would get if the link is formed. This occurs when both parties requested a link to each other. iii) Indirect connection component $(=\beta \times x)$ : the payoff a participant would get if the agent she linked with has also linked with a third agent from the group. Finally, they were informed that in the case where a participant decided not to request any links, her monetary payoff would be zero for that period.

Valuation Distribution. In the complete information setup $(\sigma=0)$, all participants were informed that, in each period, every group would receive a connection value randomly generated from the interval 50.00 to 310.00 and that any value would have an equally likely chance of being drawn. In the incomplete information setup $(\sigma>0)$, all participants were informed that every member of her group would privately receive a connection value $x_{i}$ in every period and that this value would be generated in the following manner: i) In each period and for every group, a number $x_{0}$ would be drawn randomly from 50.00 to 310.00 . Any value in this interval would have an equally likely chance of being drawn. ii) Each group member would receive a private connection value $x_{i}$ independently selected within the interval $\left[x_{0}-\sigma, x_{0}+\sigma\right]$, such that any value would have an equally likely chance of being drawn. Furthermore, participants did not know the values of $x_{0}$ or the private value of any of the other participants in their group.

Parameter Values. All participants were informed the values for alpha $(\alpha)$, beta $(\beta)$, connection cost $(c)$ and sigma $(\sigma)$ at the beginning of the session.

Minimum Capital and Payoff Procedure. Each participant received an initial balance of 9,000 points. Participants won or lost points from the total. After each round, points were added or subtracted from the initial balance. At the end of the experiment, points

\footnotetext{
${ }^{6}$ In one session of treatment 4 only 30 periods (out of 50 periods) were actually run since the network system broke down at that stage.
} 
were multiplied by $\$ 0.33$ Chilean pesos per point. The average payoff per participant for the whole session was $\$ 5,250$ (Chilean pesos), about $\$ 11.00$ US dollars.

Information Feedback. In every period, each participant observed only her own payoff, discriminated by the members of her group and discriminated by each of the three payoff components mentioned above. Throughout all of the sessions, communication among the participants was not allowed. They could not see each others' screens.
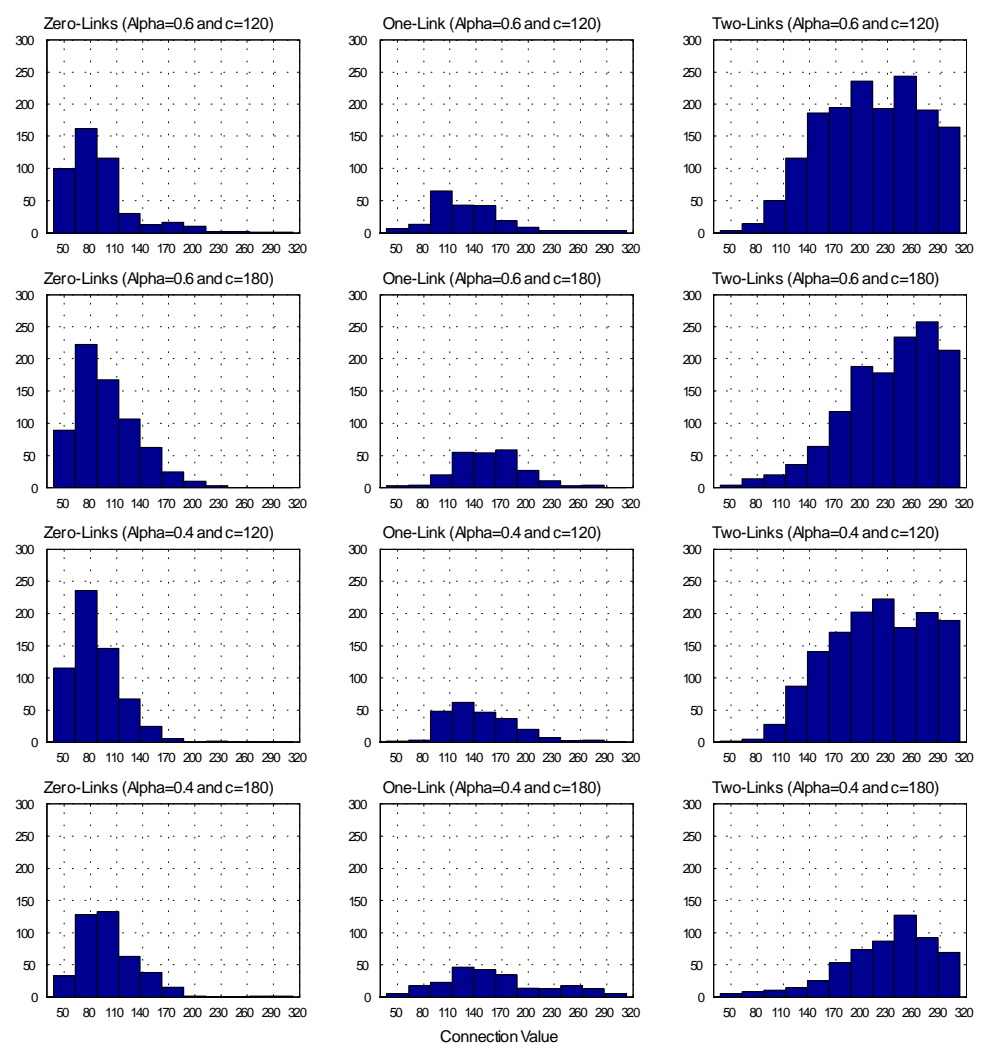

Figure 4: Histograms of Individual Decisions to $m$-link-request, for different parameter values of $\alpha$ and $c$ 


\section{Experimental Results}

We first concentrated our analysis on measuring how different proportions of connection benefits, $\alpha$, and connection costs, $c$, affect individual decisions to $m$-link-request other members of the group. Figure 4 shows a set of histograms representing the number of individual decisions to link-request with respect to the connection values, for different parameter values of $\alpha$ and $c$. For example, the first row of graphs shows, where $\alpha$ equals 0.6 and $c$ equals 120, the proportion of individuals requesting zero-links, one-link, and two-links conditional on the connection value.

Since subjects played the game multiple periods, individual's actions over time are clearly non-independent. For this reason, we initially attempted to test our hypotheses using data from only single periods. However, the results of our statistical analysis using data from single periods was inconclusive since it does not show much variation. In what follows, we instead present results of the statistical analysis using data from all experimental periods. In doing this, we use a multinomial-logit model that accounts for outcomes that can be considered independent across individuals, but not within a group of decisions made by one individual (Green, 2000 and Stata, 2007). We consider the following model for estimating the individual likelihood to link-request:

$$
\operatorname{Pr}(\text { Links }=m)=F\left(\text { Intercept }_{m \mid M}+\beta_{x, m \mid M} x_{i}+\beta_{\alpha, m \mid M} d_{\alpha}+\beta_{c, m \mid M} d_{c}+\beta_{p, m \mid M} \text { per }\right)
$$

In this model $x_{i}$ represents the connection value that each individual $i$ receives; $d_{\alpha}$ is a dummy variable that equals one when the proportion of the connection value $\alpha$ is equal to 0.4 and zero otherwise; $d_{c}$ is a dummy variable that equals one when the cost for requesting a link to another participant $c$ is equal to 180 and zero otherwise; per represents a variable for every period, treating time as a continuous variable; $F($.$) is the cumulative logistic$ distribution function; Links is equal to the response category $m$ when there are $m$-linkrequest $(m=0,1,2)$; and $M$ indicates the reference category against which other response categories are compared.

In Table 2 we present the parameter estimates representing all contrasts among all three link-request categories. A $\chi^{2}$ test indicates that the null hypothesis of all estimated coefficients equal to zero can be rejected for $p<0.0001$. Furthermore, the null hypothesis that alternative categories can be combined is rejected for the same significance threshold.

Result 1 (Comparative Static Predictions on link-request Probability) Higher connection values induce higher individual likelihood to link-request. For the same connection values, a higher connection cost, or a lower alpha, induces a higher individual 


\begin{tabular}{|c|c|c|c|}
\hline Coefficients & $\begin{array}{c}\text { One-Link } \\
\text { vs. Two-Links }\end{array}$ & $\begin{array}{c}\text { Zero-Links } \\
\text { vs. One-Link }\end{array}$ & $\begin{array}{c}\text { Zero-Links } \\
\text { vs. Two-Links }\end{array}$ \\
\hline \hline Intercept & $2.361^{* * *}$ & $4.083^{* * *}$ & $6.445^{* * *}$ \\
\hline$x_{i}$ & $(0.370)$ & $(0.325)$ & $(0.467)$ \\
\hline & $-0.025^{* * *}$ & $-0.033^{* * *}$ & $-0.059^{* * *}$ \\
\hline$d_{\alpha}$ & $(0.002)$ & $(0.003)$ & $(0.004)$ \\
\hline & $0.572^{*}$ & -0.073 & $0.499^{*}$ \\
\hline$d_{c}$ & $(0.220)$ & $(0.249)$ & $(0.201)$ \\
\hline & $1.164^{* * *}$ & 0.485 & $1.650^{* * *}$ \\
\hline$p e r$ & $(0.239)$ & $(0.259)$ & $(0.268)$ \\
\hline & $-0.009^{* *}$ & $0.019^{* * *}$ & $0.010^{*}$ \\
\hline Number of Obs. & $(0.003)$ & $(0.004)$ & $(0.004)$ \\
\hline Number of Ind. & 7,950 & & \\
\hline Log Likelihood & -3792.5 & & \\
\hline
\end{tabular}

${ }^{*}: p<0.05,{ }^{* *}: p<0.01$, and ${ }^{* * *}: p<0.001$.

Note: Numbers in parentheses below each coefficient represent the coefficient standard error.

Table 2: Multinomial Logit Model Results for Individual Link-Request Probability, for Different Parameter Values of alpha and c

likelihood to zero-links and to one-link requests, and a lower individual likelihood to twolinks request. Finally, for the same connection values, there is over time a higher individual likelihood to link-request either everyone (two-links) or no-one (zero-links).

Support for Result 1 Connection value coefficients $\left(\beta_{x, 1 \mid 2}, \beta_{x, 0 \mid 1}\right.$, and $\left.\beta_{x, 0 \mid 2}\right)$ are negative, as expected, and significant. This means that the individual likelihood to linkrequest a larger number of agents increases as the connection value increases. For the contrast of zero-links versus two-links request, the coefficient associated with a higher connection cost $\left(\beta_{c, 0 \mid 2}\right)$ is positive, as expected, and significant. This is also true for the coefficient associated with a lower alpha $\left(\beta_{\alpha, 0 \mid 2}\right)$. This means that a higher connection cost, or a lower alpha, induces a lower individual likelihood to two-links request and a higher individual likelihood to zero-links request. For the contrast of one-link versus two-links request, the coefficient associated with a higher connection cost $\left(\beta_{c, 1 \mid 2}\right)$ is positive and significant, as is the coefficient associated with a lower alpha $\left(\beta_{\alpha, 1 \mid 2}\right)$. Since an increment of the connection cost, or a reduction in alpha, shrinks the upper region of dominance, it might be more difficult for agents to coordinate when to request two-links instead of one-link. Our estimation also shows that the time period coefficients $\left(\beta_{p, 1 \mid 2}\right)$ and $\left(\beta_{p, 0 \mid 1}\right)$ are both significant. The negative sign of the first coefficient $\left(\beta_{p, 1 \mid 2}\right)$ and the positive sign of the second coefficient $\left(\beta_{p, 0 \mid 1}\right)$ imply that, over time, participants are willing to 
play homogeneous strategies more often than heterogeneous strategies, requesting links to either everyone or no-one, rather than just one member of their own group.

Figure 5 shows for each parameter value of $\alpha$ and $c$ the estimated individual linkrequest probabilities with respect to different connection values. Thus, the first row of graphs shows, for $\alpha$ equal to 0.6 and $c$ equal to 120 , the probability distributions of requesting zero-links, one-link, and two-links. Each graph shows two lines representing each of the probability distributions for the first and last periods.

Now we compare the predicted switching thresholds, $k^{*}$ and $\underline{x}$, and the estimated mean switching thresholds $\widehat{k}$, and check how the estimated switching thresholds change in terms of our comparative static predictions. We estimate the mean switching threshold for which the probability of two-links request is equal to the probability of zero-links request. This value indicates the mean threshold above which individuals are willing to switch between these two homogeneous strategies. That is, switching from zero-links to two-links request. To calculate $\widehat{k}$ and its standard error, we implement the delta method using the estimated logistic distributions (Green, 2000). In Table 3 we report the estimated mean switching threshold for each session, jointly with the estimated standard errors. In the same table, we present the equilibrium switching threshold $k^{*}=(4 c) /(2+4 \alpha+(4 / 3) \beta)$ predicted by global games and the equilibrium switching threshold $\underline{x}=c /(1+\alpha+\beta)$ predicted by $S N E$. In Figure 6 we present the estimated mean switching thresholds for each treatment, within the $95 \%$ confidence intervals.

Result 2 (Equilibrium Selection on Switching Threshold) Estimated mean switching threshold for different alphas and connection costs do not differ from the global games predicted switching thresholds, when the dominance regions are intersected by the connection value distribution support. If the upper dominance region is above the upper support of the distribution (treatment 4), the estimated mean switching threshold is between the global games and the strong Nash predictions.

Support for Result 2 It can be appreciated in Figure 6 that the predicted $k^{*}$ is within two standard errors of the estimated mean switching thresholds for Treatments 1, 2 and 3. For Treatment 4, however, the estimated threshold is between the predicted values of $k^{*}$ and $\underline{x}$. This outcome could be a result of the fact that for this treatment the upper bound of the payoff dominance region, $\bar{x}(=c / \alpha)$, is above the upper bound of the connection value distribution. If the upper dominance region does not exist, the global games approach does not guarantee a uniqueness result, because the process of iterated elimination cannot be made.

Result 3 (Comparative Statics on Switching Thresholds) An increment in connection costs induces an increment in the estimated mean switching thresholds. Mean- 

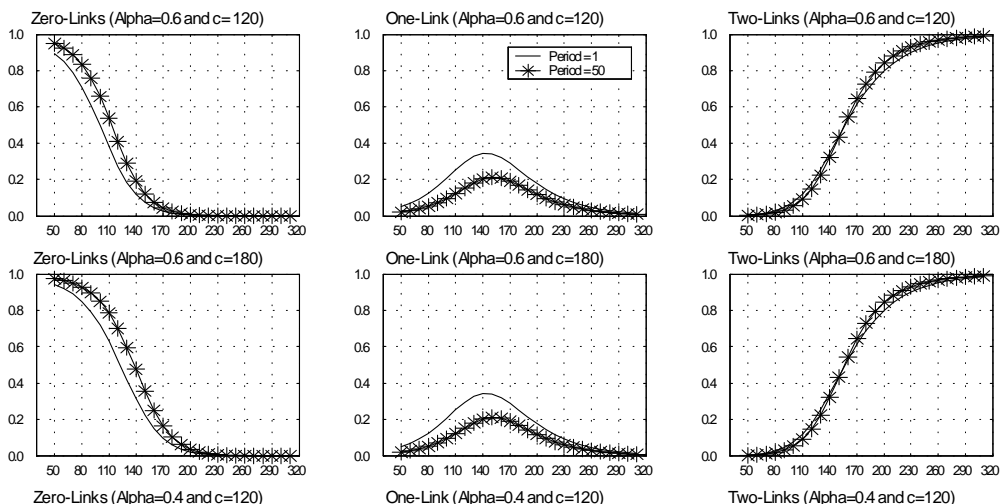

Two-Links (Apha $=0.6$ and $=180$ )
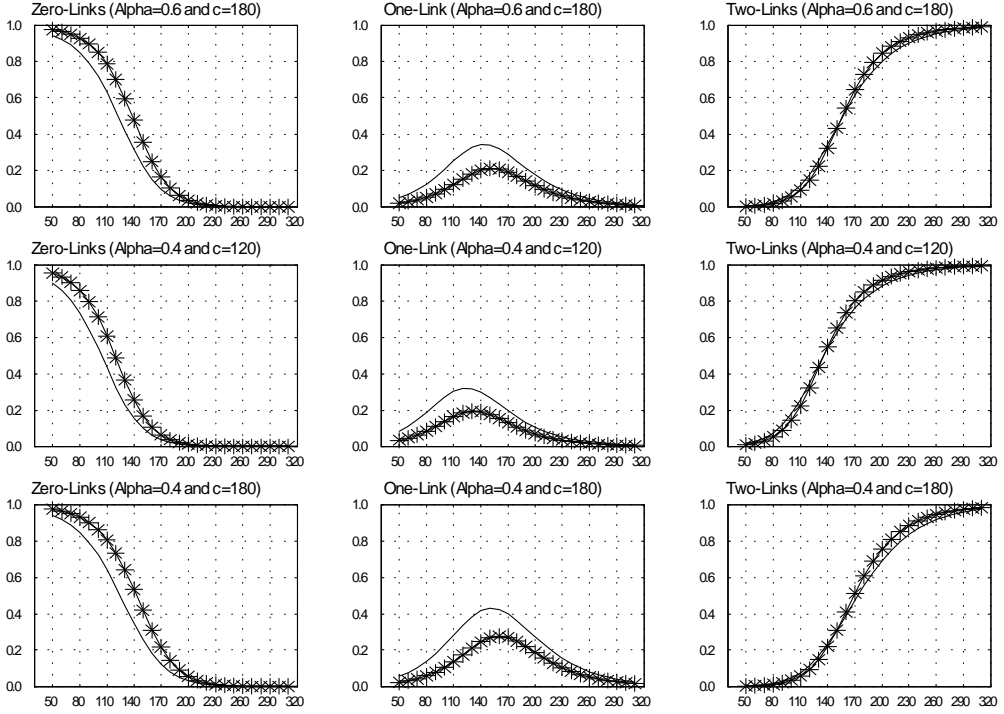

Figure 5: Estimated Link-Request Probability Distributions, for Different Parameter Values of $\alpha$ and $c$

\begin{tabular}{|c|c|c|c|c|c|}
\hline Treatment & Session & $\widehat{k}$ & $k^{*}$ & $\underline{x}$ & $\widehat{s e}$ \\
\hline \hline \multirow{3}{*}{$(\alpha, c)=(0.6,120)$} & 1 & 116 & 103 & 67 & 4.9 \\
\cline { 2 - 6 } & 2 & 109 & 103 & 67 & 4.6 \\
\cline { 2 - 6 } & 3 & 116 & 103 & 67 & 2.9 \\
\hline \multirow{3}{*}{$(\alpha, c)=(0.6,180)$} & 4 & 154 & 154 & 100 & 12.9 \\
\cline { 2 - 6 } & 5 & 139 & 154 & 100 & 5.3 \\
\cline { 2 - 6 }$(\alpha, c)=(0.4,120)$ & 6 & 138 & 154 & 100 & 5.7 \\
\cline { 2 - 6 } & 7 & 126 & 124 & 75 & 5.2 \\
\cline { 2 - 6 } & 9 & 120 & 124 & 75 & 6.5 \\
\hline \multirow{3}{*}{$(\alpha, c)=(0.4,180)$} & 11 & 142 & 124 & 75 & 4.0 \\
\cline { 2 - 6 } & 1157 & 186 & 113 & 6.4 \\
\hline \multirow{2}{*}{$(\alpha)$} & & & & & \\
\hline
\end{tabular}

Table 3: Estimated Mean Switching Threshold and its Standard Error 


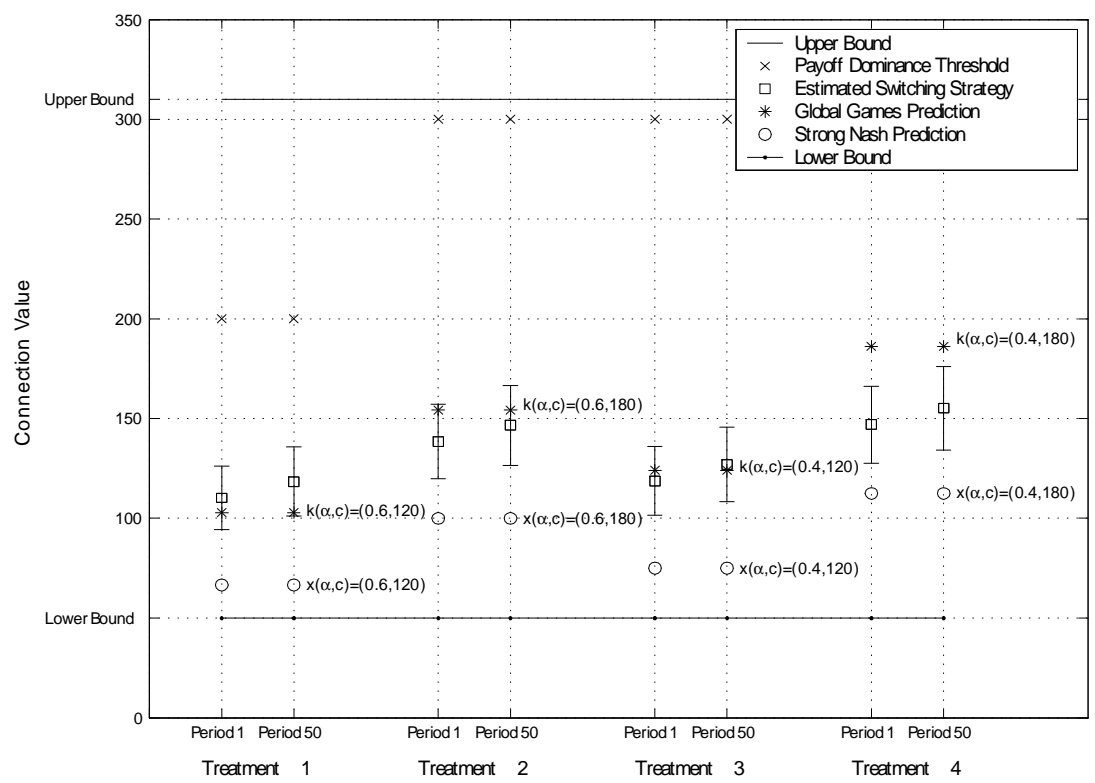

Figure 6: Predicted and Estimated Mean Switiching Strategies (First and Last Periods)

while, there is not enough evidence that a reduction in alpha induces a significant increment in the estimated mean switching thresholds.

Support for Result 3 It can be seen in Figure 6 that an increment of $c$ induces a significant increment of the estimated mean switching threshold. A $t$-test for differences in means at every period indicates that the null hypotheses that estimates of $\widehat{k}$ for different connection costs are equal can be rejected for $p<0.001$. In the same fashion, a reduction of alpha does not induce an increment of $\widehat{k}$. A $t$-test for differences in means at every period indicates that the null hypotheses that estimates of $\widehat{k}$ for different alpha values are equal cannot be rejected for $p<0.05$.

We now concentrate our analysis on measuring how different alphas and connection costs $c$ have an affect on the final structure of the network in the link-formation game. Similar to our individual behavior analysis, the results of our statistical analysis using data only from the first period were inconclusive, as were the results using data from the last period. In what follows, we present results of the statistical analysis involving data from all experimental periods. In doing this, we use a multinomial-logit model that accounts for outcomes that can be considered independent across groups. We consider the following model for estimating network structure likelihood:

$$
\operatorname{Pr}(\text { Structure }=n)=F\left(\text { Intercept }_{n \mid N}+\delta_{x, n \mid N} x+\delta_{\alpha, n \mid N} d_{\alpha}+\delta_{c, n \mid N} d_{c}+\delta_{p, n \mid N} \text { per }\right)
$$


In this model $x_{0}$ represents the connection value each group receives; $d_{\alpha}$ is a dummy variable that equals one when the proportion of the connection value $\alpha$ is equal to 0.4 and zero otherwise; $d_{c}$ is a dummy variable that equals one when the cost for requesting a link to another participant $c$ is equal to 180 and zero otherwise; per represents a variable for every period, treating time as a continuous variable; $F($.$) is the cumulative logistic$ distribution function; Structure is equal to the response category $n$ when there are $n$ connections formed. $n$ will equal to zero if there is an empty network, one if there is a one-connection network, two if there is a two-connections network, and three if there is a complete network. $N$ indicates the reference category against which other response categories are compared.

In Table 4, we present the parameter estimates representing all contrasts among all four categories. A $\chi^{2}$ test indicates that the null hypothesis of all estimated coefficients equal to zero can be rejected for $p<0.0001$. Furthermore, the null hypothesis that alternative categories can be combined is rejected for the same significance threshold.

Results 4 (Comparative Statics on Network Structure) Higher connection values induce a higher likelihood for more connected networks. For the same connection values, a higher connection cost induces a lower likelihood for more connected networks. Meanwhile, a lower alpha induces a lower likelihood for complete networks against any other network structure for the same connection values. Finally, over time and for the same connection values there is a lower likelihood for two-connections and one-connection networks and a higher likelihood for complete and empty networks.

Support for Result 4 Connection value coefficients $\left(\delta_{x, 2 \mid 3}, \delta_{x, 1 \mid 2}\right.$, and $\left.\delta_{x, 0 \mid 1}\right)$ were negative, as expected, and significant. This means that the likelihood for more connected networks increases as the connection value increases. All coefficients associated with a higher connection cost $\left(\delta_{c, 2 \mid 3}, \delta_{c, 1 \mid 2}\right.$, and $\left.\delta_{c, 0 \mid 1}\right)$ are all positives and significant. This indicates that for the whole support of connection value distribution, the proportion of less connected networks increases when connection cost increases. Coefficients associated with a lower value of alpha for the contrast of zero, one and two-connections networks against complete networks $\left(\delta_{\alpha, 0 \mid 3}, \delta_{\alpha, 1 \mid 3}\right.$ and $\left.\delta_{\alpha, 2 \mid 3}\right)$ are all positive and significant. This indicates that for a lower value of alpha, the proportion of complete networks decreases and the proportion of zero, one and two-connections networks increases for the whole support of the connection value distribution. Our estimation shows that time period coefficients $\delta_{p, 2 \mid 3}, \delta_{p, 1 \mid 3}, \delta_{p, 0 \mid 2}$ and $\delta_{p, 0 \mid 1}$ are all individually significant. The negative sign of the first two coefficients $\left(\delta_{p, 2 \mid 3}\right.$ and $\left.\delta_{p, 1 \mid 3}\right)$ and the positive sign of the last two coefficients $\left(\delta_{p, 0 \mid 2}\right.$ and $\left.\delta_{p, 0 \mid 1}\right)$ imply that, over time, the proportion of complete and empty networks tend to increase. This can be seen as a result of the increasing individual likelihood to play homogeneous instead of heterogeneous strategies over time. 


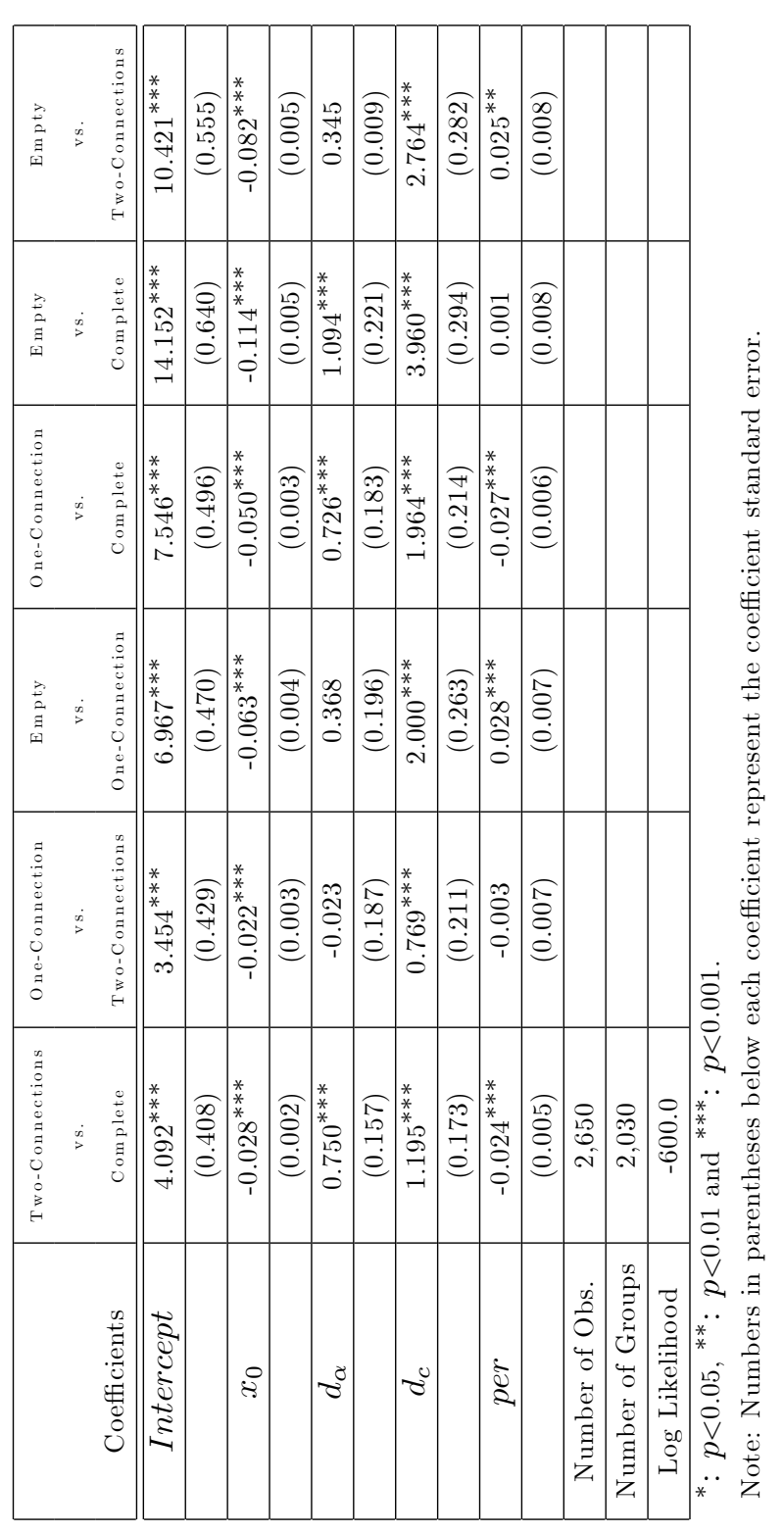

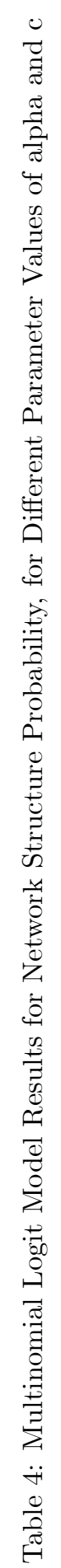




\begin{tabular}{|c|c|c|c|}
\hline Coefficients & $\begin{array}{c}\text { One-Link } \\
\text { vs. Two-Links }\end{array}$ & $\begin{array}{c}\text { Zero-Links } \\
\text { vs. One-Link }\end{array}$ & $\begin{array}{c}\text { Zero-Links } \\
\text { vs. Two-Links }\end{array}$ \\
\hline \multirow[t]{2}{*}{ Intercept } & $2.839^{* * *}$ & $5.359^{* * *}$ & $8.198^{* * *}$ \\
\hline & $(0.542)$ & $(0.421)$ & $(0.719)$ \\
\hline \multirow[t]{2}{*}{$x_{i}$} & $-0.024^{* * *}$ & $-0.043^{* * *}$ & $-0.067^{* * *}$ \\
\hline & $(0.003)$ & $(0.004)$ & $(0.005)$ \\
\hline \multirow[t]{2}{*}{$d_{\sigma_{10}}$} & $0.695^{*}$ & -0.016 & $0.678^{*}$ \\
\hline & $(0.318)$ & $(0.341)$ & $(0.319)$ \\
\hline \multirow[t]{2}{*}{$d_{\sigma_{50}}$} & $1.160^{*}$ & $-1.071^{*}$ & 0.089 \\
\hline & $(0.539)$ & $(0.503)$ & $(0.533)$ \\
\hline \multirow[t]{2}{*}{ per } & $-0.014^{* *}$ & $0.015^{* *}$ & 0.001 \\
\hline & $(0.004)$ & $(0.005)$ & $(0.006)$ \\
\hline Number of Obs. & 4,500 & & \\
\hline Number of Ind. & 90 & & \\
\hline Log Likelihood & -2177.6 & & \\
\hline
\end{tabular}

Note: Numbers in parentheses below each coefficient represent the coefficient standard error.

Table 5: Multinomial Logit Model Result for Individual Link Request Probability for different parameter values of sigma

\subsection{Robustness of the Global Games Predictions}

We now focus on measuring how different levels of perturbation, $\sigma$, in the payoff function affects individual decisions to request a link.

Since subjects played the game multiple periods, individual's actions over time are clearly non-independent. We proceed as in the first estimation, considering the following model for estimating the individual likelihood to link-request:

$\operatorname{Pr}($ Links $=m)=F\left(\right.$ Intercept $_{m \mid M}+\gamma_{x, m \mid M} x_{i}+\gamma_{\sigma_{10}, m \mid M} d_{\sigma_{10}}+\gamma_{\sigma_{50}, m \mid M} d_{\sigma_{50}}+\gamma_{p, m \mid M}$ per $)$

In this model $x_{i}$ represents the connection value that individual $i$ receives; $d_{\sigma_{10}}$ and $d_{\sigma_{50}}$ are dummy variables when the perturbation level $\sigma$ is equal to 10 and 50 , respectively; per represents a variable for every period, treating time as a continuous variable; $F($.) is the cumulative logistic distribution function; Links is equal to the response category $m$ when there are $m$-link-request $(m=0,1,2)$; and $M$ indicates the reference category against which other response categories are compared.

In Table 5, we present the parameter estimates representing all contrasts among all three link-request categories. A $\chi^{2}$ test indicates that the null hypothesis of all the esti- 
mated coefficients are equal to zero can be rejected for $p<0.0001$. Furthermore, the null hypothesis that alternatives categories can be combined is rejected for the same significance threshold.

Result 5 (Perturbation Effect on link-request Probability) Higher connection values induce a higher individual likelihood to link-request. For the same connection values, larger perturbations induce lower individual likelihood to two-links request. Finally, for the same connection values, there is over time a higher individual likelihood to link-requests for either everyone or no-one.

Support for Result 5 Connection value coefficients $\left(\gamma_{x, 1 \mid 2}, \gamma_{x, 0 \mid 1}\right.$, and $\left.\gamma_{x, 0 \mid 2}\right)$ were negative, as expected, and significant. For the perturbation size equal to fifty, coefficients for one-link requests versus zero and two-links requests $\left(\gamma_{\sigma_{50}, 1 \mid 2}\right.$ and $\gamma_{\sigma_{50}, 0 \mid 1}$, respectively) are both significant. A positive sign on the first coefficient and a negative sign on the second indicate that individuals increase the likelihood to one-link request as perturbation size increases. The same is observed for the coefficient associated with the perturbation size equal to ten for the contrast between the one-link versus the two-links request $\left(\gamma_{\sigma_{10}, 1 \mid 2}\right)$. However, from the contrast between the one-link versus the zero-links request $\left(\gamma_{\sigma_{10}, 0 \mid 1}\right)$ the coefficient is not significant. The other coefficient significance arises for the contrast between the zero-links versus the two-links request $\left(\gamma_{\sigma_{10}, 0 \mid 2}\right)$, though we have no reasonable explanation for this result. Similar to our result in the previous section, the negative sign of the first time period coefficient $\left(\gamma_{p, 1 \mid 2}\right)$ and the positive sign of the second time period coefficient $\left(\gamma_{p, 0 \mid 1}\right)$ implies that over time participants were willing to play more often homogeneous instead of heterogeneous strategies, requesting links to either everyone or no-one, rather than to just one member of their own group.

Figure 7 shows for each parameter value of sigma the estimated individual link-request probabilities with respect to different connection values. Thus, the first row of graphs shows for sigma equal to zero the probability distributions of requesting zero-links, onelink, and two-links, respectively. Each graph shows two lines representing each of the probability distributions for the first and last periods.

Result 6 (Perturbation Effect on Switching Threshold and Coordination) Estimated mean switching threshold for the different perturbation levels do not differ from the global games predicted threshold. The estimated mean switching threshold for sigma equal to zero is similar at every period to those for sigma greater than zero.

Support for Result 6 In Figure 8 we present the estimated switching threshold of period one and period fifty with $95 \%$ confidence intervals, jointly with the equilibrium 

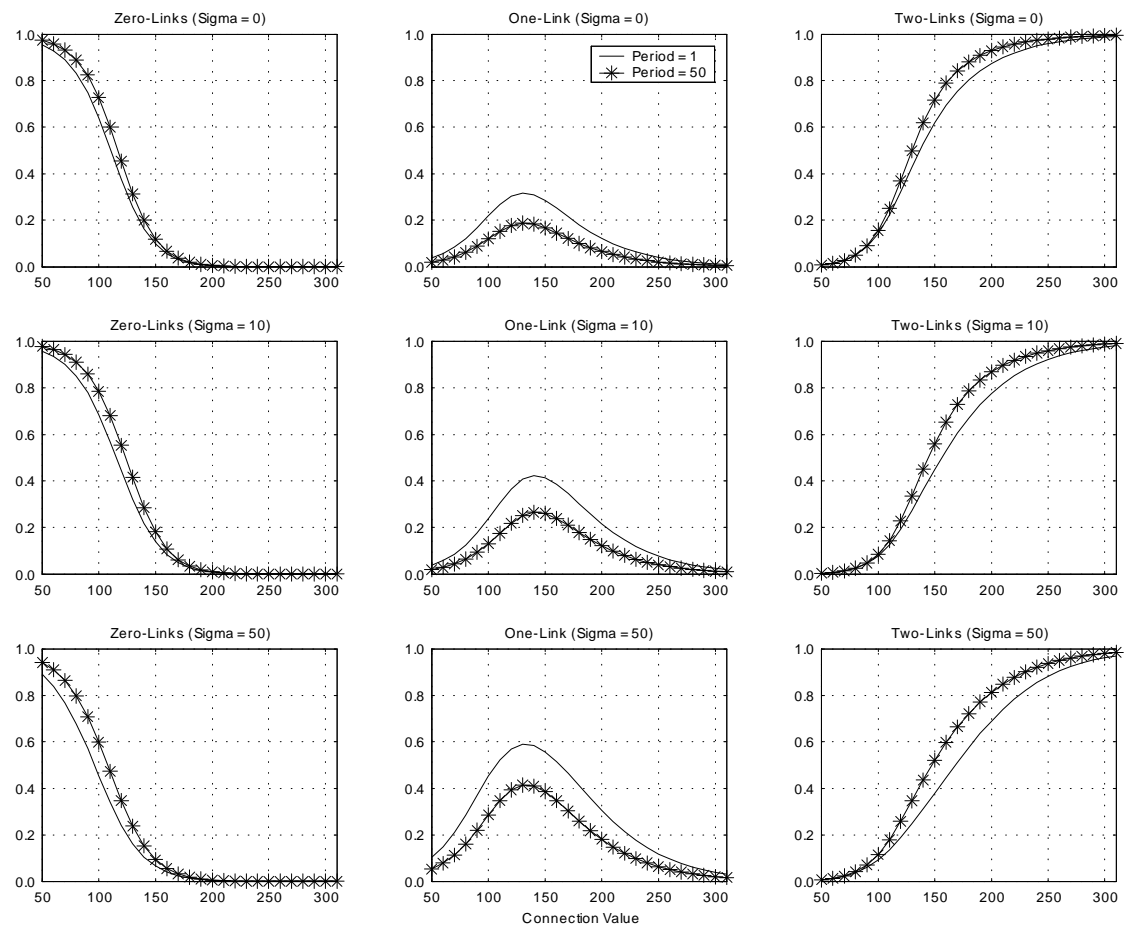

Figure 7: Estimated Link-Request Probability Distributions for different parameter values of sigma 


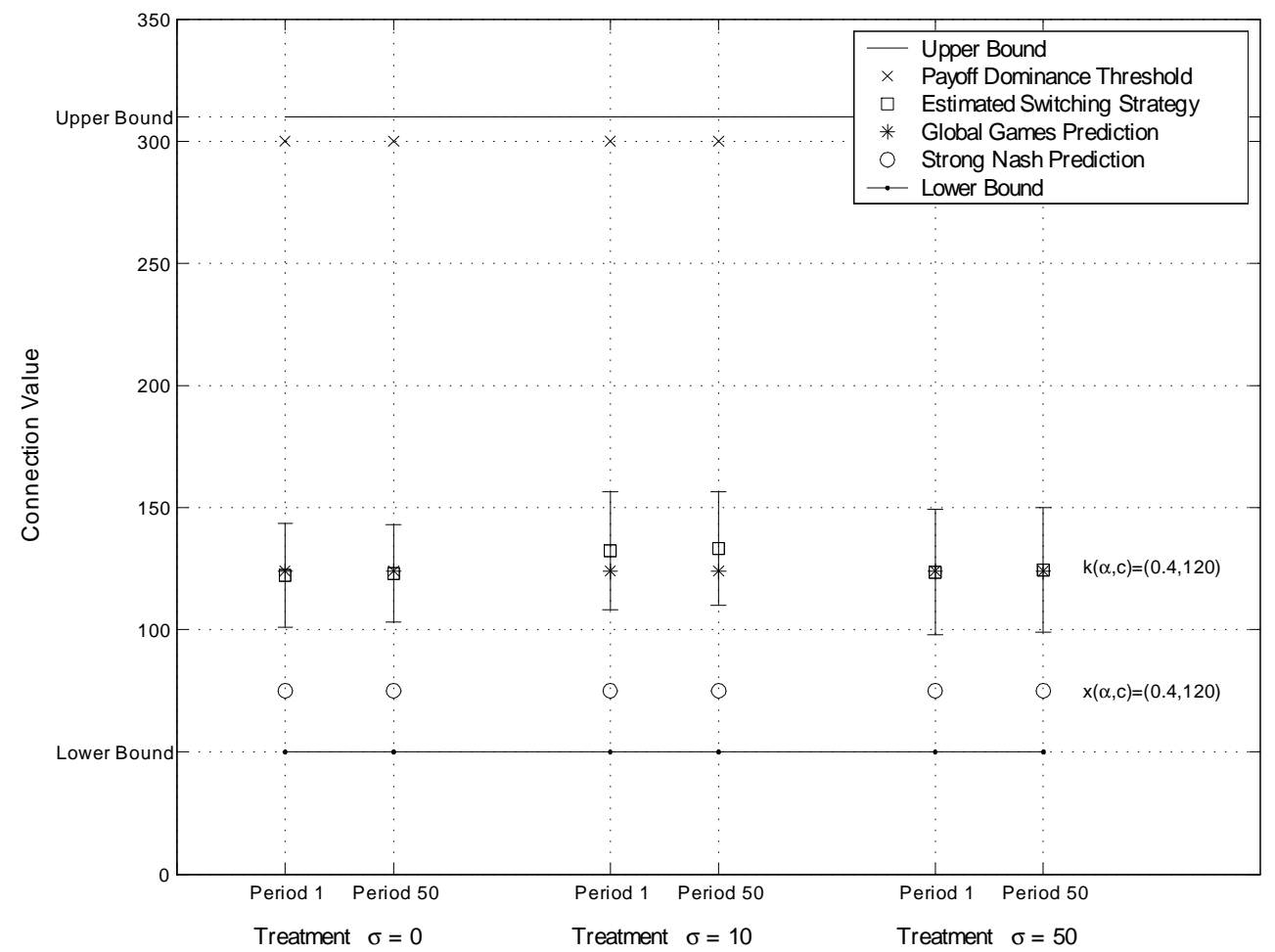

Figure 8: Predicted and Estimated Switching Strategies, for Different Parameter Values of Sigma (First and Last Periods)

switching threshold $k^{*}=(4 c) /(2+4 \alpha+(4 / 3) \beta)$ predicted by global games and the equilibrium switching threshold $\underline{x}=c /(1+\alpha+\beta)$ predicted by $S N E$. It can be appreciated in this Figure that the predicted $k^{*}$ is within two standard errors of the estimated switching strategies $\widehat{k}$, for all values of sigma. Additionally, a $t$-test for differences in means at every period indicates that the null hypotheses that estimates of $\widehat{k}$ for different values of sigma are equal cannot be rejected for a $p<0.05$.

\section{Conclusion}

In this paper, we compare experimentally the accuracy of global game and alternative cooperative refinements theories, such as $S N E$ and $C P N E$, that are used to predict network configurations in a link-formation game. Our results indicate that the global games approach does significantly better than the cooperative games solutions pointwise predicting the switching thresholds above which individuals are willing to link other players in the net.

We also check for robustness of these results by testing the comparative static predic- 
tions derived from changes in the parameter values of the payoff function and by introducing small amounts of perturbation in the payoff function. Our results indicate, first, that higher connection values induce a higher proportion of agents requesting one or two links and, consequently, a higher likelihood of more connected networks. Second, that higher connection costs, given the support of connection values, induce a lower proportion of agents requesting a link to the other two members of their groups and, consequently, a lower proportion of fully connected networks. Third, that lower values of alpha induce a lower likelihood for complete networks, though at an individual level, it does not have an effect on the proportion of agents expressing their wish to be connected to. Fourth, for the same connection values, there is over time a higher proportion of agents willing to request a link to either everybody or nobody and, consequently, a lower proportion of partially connected networks. And, finally, different levels of noise do not significantly affect the selected switching threshold, which is accurately predicted by the global games approach.

Even though our results seem to be robust, the experiment is limited to a specific payoff function, number of players, and environment of non-repeated interaction. It will be interesting to see, to what degree, the global game approach preserve its power for different game structures.

\section{References}

Aumann, R. (1959) "Acceptable Points in General Cooperative n-Person Games", in H. W. Kuhn and R. D. Luce, eds., Contributions to the Theory of Games IV, Princeton: Princeton University Press, 287-324.

Bala, V. and S. Goyal (2000a) "A Noncooperative Model of Network Formation," Econometrica, 68: 1181-1229.

Cabrales, A., R. Nagel, and R. Armenter (2007) "Equilibrium Selection Through Incomplete Information in Coordination Games: An Experimental Study" forthcoming in Experimental Economics.

Callander, S. and C.R. Plott (2005) "Principles of network development and evolution: an experimental study," Journal of Public Economics, 89: 1469-1495.

Deck, C. and C. Johnson (2004) "Link Bidding in a Laboratory Experiment," Review of Economic Design, 8: 359-372.

Dutta, B. and M. Jackson (2003) "On the formation of Networks and Groups," in Models of the Strategic Formation of Networks and Groups, , edited by Bhaskar Dutta and Matthew O. Jackson, Springer-Verlag, Heidelberg.

Dutta, B. and S. Mutuswami (1997) "Stable Networks," Journal of Economic Theory, $76,322-344$.

Dutta, B., A. van den Nouweland and S. Tijs (1998) "Link Formation in Cooperative Situations," International Journal of Game Theory, 27, 245-256. 
Falk, A. and M. Kosfeld (2005) "It's all about Connections: Evidence on Network Formation," mimeo, University of Zurich.

Fafchamps, M. and Lund, S. (2003) "Risk-sharing networks in rural Philippines," Journal of Development Economics, Elsevier, vol. 71(2), pages 261-287, August.

Frankel, D., S. Morris and A. Pauzner (2003) "Equilibrium Selection in Global Games with Strategic Complementarities., Journal of Economic Theory, 108, 1-44.

Green, W. H. (2000) Econometric Analysis, Prentice Hall, NJ.

Harrison, R. and R. Muñoz (2007) "Stability and Equilibrium Selection in Link Formation Games," forthcoming in Economic Theory.

Heinemann, F., R. Nagel and P. Ockenfels (2004) "The Theory of Global Games on Test: Experimental Analysis of Coordination Games with Public and Private Information," Econometrica, 72 (5), 1583-1599.

Jackson, M. (2003) "The Stability and Efficiency of Economic and Social Networks," in Advances in Economic Design, edited by Murat Sertel and Semih Koray, Springer-Verlag, Heidelberg.

Jackson, M. (2004) "A Survey of Models of Network Formation: Stability and Efficiency," in Group Formation in Economics; Networks, Clubs and Coalitions, edited by Gabrielle Demange and Myrna Wooders, Cambridge University Press, Cambridge UK.

Jackson, M. and A. van den Nouweland (2005) "Strongly Stable Networks," Games and Economic Behavior, 51(2), 420-444.

Jackson, M. and A. Wolinsky (1996) "A Strategic Model of Social and Economic Networks," Journal of Economic Theory, 71, 44-74.

Montgomery, J. M. (1991) "Social Networks and Labor-Market Outcomes: Toward an Economic Analysis," American Economic Review, 81, 5, pp. 1408-1418

Morris, S. and H. Shin (2002) "Global Games: Theory and Applications," in Advances in Economics and Econometrics (Proceedings of the Eight World Congress of the Econometric Society), edited by M. Dewatripont, L. Hansen, and S. Turnosky. Cambridge, England: Cambridge University Press.

Myerson, R. (1991) Game Theory: Analysis of Conflict, Harvard University Press: Cambridge, MA.

Riordan, J. (1968) Combinatorial Identities, Wiley Series in Probability and Mathematical Statistics.

Slikker M. and A. van den Nouweland (2000) "Network Formation Models with Costs for Establishing Links", Review of Economic Design, 5, 333-362.

Shurchkov, O. (2006) "Coordination and Learning in Dynamic Global Games: Experimental Evidence," mimeo MIT. 


\section{A Experimental Instructions}

The following is the verbatim translation (from Spanish into English) of experimental instructions administered to subjects at PUC (the Spanish original is available from the authors upon request).

\section{A.1 Instructions (Treatment $\sigma=0$ )}

This is an experiment about decision-making. Funding for this research has been provided by several academic institutions. Instructions are simple and if you follow them carefully and make good decisions, you can earn a CONSIDERABLE AMOUNT OF MONEY, which will be PAID WITH A CHECK that you can immediately cash in the Bank office inside the campus.

General Proceedings

1. In this experiment you will participate as a member of a group of agents with the opportunity to link each other. The experiment consists of 60 periods: 10 practice periods, which won't count for your final payoff, and 50 periods to be played for money.

2. At the beginning of every period, the computer will randomly form groups of three participants. Each of you will form part of a new group in each of the following periods. You will always be identified as agent 1 in the computer screen. Furthermore, you will never know who you are grouped with in any given period.

3. In every period, you have to decide whether to request a link to each member of your group.

4. In every period, each member of a group will receive a CONNECTION VALUE $\left(X_{0}\right)$. This CONNECTION VALUE will be identical for all members of the same group and it will be generated randomly by the computer from the interval 50 to 310 points. Any value in this interval will have an equally likely chance of being drawn and it will be generated independently from previous periods.

For example, in each period, the computer will randomly form groups of three participants and it will assign a random CONNECTION VALUE, $X_{0}$, to each group. Suppose that a particular group gets an $X_{0}$ equal to 200 points. Therefore, all members of this group will have a CONNECTION VALUE of 200. That is, the CONNECTION VALUE of agent $1\left(X_{1}\right)$ will be equal to that of agent $2\left(X_{2}\right)$ and to that of agent $3\left(X_{3}\right)$.

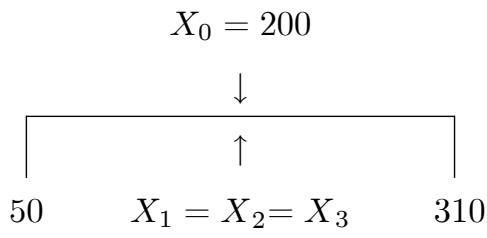

The members of each group should decide whether to connect the other members in their group.

5. Then, you and every member of your group will have to decide whether to request zero, one, or two links. The connection costs and the monetary payoffs that you receive will depend on your decisions to link-request other members of your group and the decisions of the other members of your group to link-request you and among themselves. The costs and the monetary 
payoffs for each link-request you decide to make will be the sum of the following three payoff components:

5.1 Independent connection payoff: For each member of your group you decide to link-request, you will get the payment of:

\section{ALPHA $\times X_{i}-$ CONNECTION COST}

For each agent you decide to NOT link-request, you will get zero as payoff, even if any other agent has decided to link-request you.
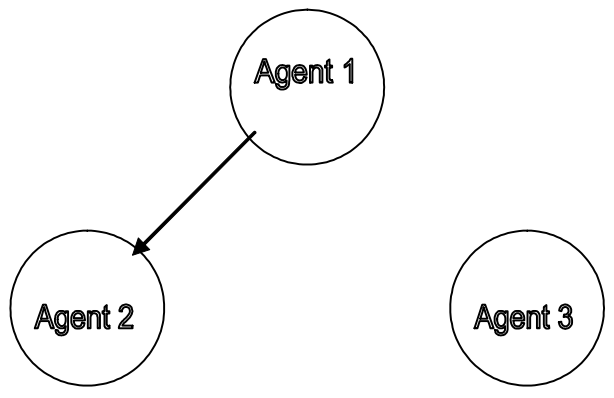

For example, if agent 1 decides to link-request agent 2 and to NOT link-request agent 3, as in the figure above, then agent 1 will get the amount of $A L P H A \times X_{1}-C O N N E C T I O N$ $C O S T$ for link-request agent 2 and zero for NOT link-request agent 3.

5.2. Direct connection payoff: If the agent you have link-request decides to link-request you in return, then you will get the amount of your CONNECTION VALUE $\left(X_{i}\right)$ as an additional payoff.
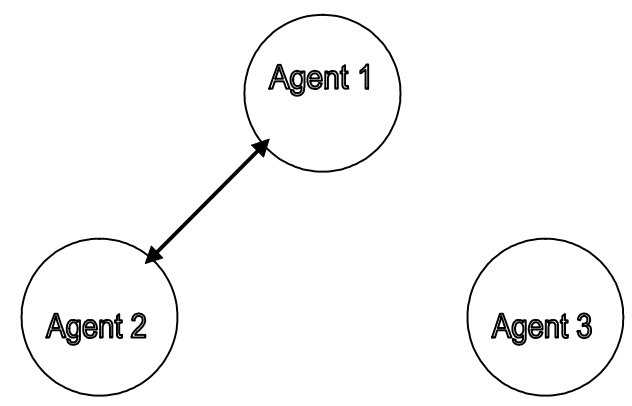

For example, if agents 1 and 2 decide to link-request each other, as in figure above, then agent 1 will get the amount of $A L P H A \times X_{1}-C O N N E C T I O N C O S T$ plus the amount of her $X_{i}$ : That is, $(1+A L P H A) \times X_{1}-C O N N E C T I O N C O S T$. The same will happen with agent 2 .

5.3. Indirect connection payoff: If the agent you have established a direct connection has also established a direct connection with the third agent of your group, you will get the amount of $B E T A \times X_{1}$ as an additional payoff. 


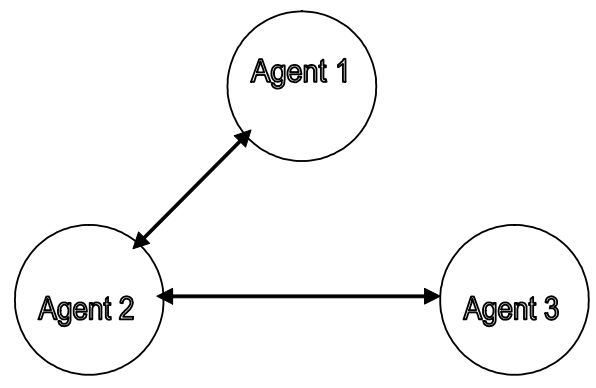

For example, if agents 1 and 2 and agents 2 and 3 decide to link-request each other, as in figure above, then agent 1 will get the sum of all three payoff components: Independent, Direct and Indirect connection payoffs. That is, agent 1 will receive the amount of $A L P H A \times X_{1}-$ $C O N N E C T I O N C O S T+X_{1}+B E T A \times X_{1}$ : That is, $(1+A L P H A+B E T A) \times X_{1}-$ CONNECTION COST

Notice that agent 1 will get her payoff for having an indirect connection with agent 3 through agent 2 even if she has an independent connection with agent 3 . Likewise, the amount an agent can get might be negative or positive depending on her CONNECTION VALUE.

According to the last figure above, agent 1 would get the following payoff.

Payoff Table for Agent 1

\begin{tabular}{|c|c|c|c|c|c|}
\hline Agent & $\begin{array}{l}\text { Independent } \\
\text { Connection }\end{array}$ & $\begin{array}{c}\text { Direct } \\
\text { Connection }\end{array}$ & $\begin{array}{c}\text { Indirect } \\
\text { Connection }\end{array}$ & $\begin{array}{c}\text { Total Payoff } \\
\text { per Connection }\end{array}$ & $\begin{array}{l}\text { Total } \\
\text { Payoff }\end{array}$ \\
\hline Agent 2 & $\begin{array}{c}A L P H A \times X_{1}- \\
C . C O S T\end{array}$ & $X_{1}$ & $B E T A \times X_{1}$ & $\begin{array}{c}A L P H A \times X_{1}+ \\
X_{1}+B E T A \times X_{1}- \\
\text { C.COST }\end{array}$ & $\begin{array}{c}A L P H A \times X_{1}+ \\
X_{1}+\end{array}$ \\
\hline Agent 3 & 0 & 0 & 0 & 0 & $\begin{array}{c}B E T A \times X_{1}- \\
C . C O S T\end{array}$ \\
\hline
\end{tabular}




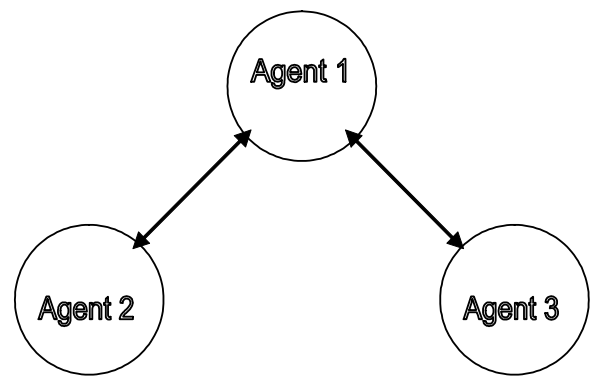

Exercise:

Just for clarification and as an exercise, fill the table indicating how much agent 1 will get given the following graph of connections.

Fill up the following table assuming that the individual CONNECTION VALUE is $X_{1}$.

\begin{tabular}{|c|c|c|c|c|c|}
\hline Agent & $\begin{array}{c}\text { Independent } \\
\text { Connection }\end{array}$ & $\begin{array}{c}\text { Direct } \\
\text { Connection }\end{array}$ & $\begin{array}{c}\text { Indirect } \\
\text { Connection }\end{array}$ & $\begin{array}{c}\text { Total Payoff } \\
\text { per Connection }\end{array}$ & $\begin{array}{c}\text { Total } \\
\text { Payoff }\end{array}$ \\
\hline Agent 2 & & & & & \\
\hline Agent 3 & & & & & \\
\hline
\end{tabular}

6. Initial Capital Balance, Capital Accumulation, and Minimum Capital Balance

Each agent will begin the experiment with a capital balance of 9000 points. Total gains (or loses) in each period will be added (or subtracted) from the previous accumulated capital. If your capital balance in any period is lower than 4500 point, you will not be able to continue participating in the experiment. You will get your capital balance and will have to leave the room.

7. Payment Procedure

Your final payoff will be equivalent to the final capital balance multiplied by 0.33 plus your participation fee. You will receive the amount in check that you can immediately cash at the bank office inside the campus.

¿Are there any questions?

\section{A.2 Instructions (Treatment $\sigma>0$ )}

This is an experiment about decision-making. Funding for this research has been provided by several academic institutions. Instructions are simple and if you follow them carefully and make good decisions, you can earn a CONSIDERABLE AMOUNT OF MONEY, which will be PAID WITH A CHECK that you can immediately cash in the Bank office inside the campus.

General Proceedings

1. In this experiment you will participate as a member of a group of agents with the opportunity to link each other. The experiment consists of 60 periods: 10 practice periods, which won't count for your final payoff, and 50 periods to be played for money. 
2. At the beginning of every period, the computer will randomly form groups of three participants. Each of you will form part of a new group in each of the following periods. You will always be identified as agent 1 in the computer screen. Furthermore, you will never know who you are grouped with in any given period.

3. In every period, you have to decide whether to request a link to each member of your group.

4. In every period, the computer will randomly generate a value $X_{0}$ for each group, from the interval 50 to 310 points. Any value in this interval will have an equally likely chance of being drawn and it will be generated independently from previous periods. Then each member of a group will receive a CONNECTION VALUE $\left(X_{i}\right)$, which will be randomly generated from the interval $X_{0}-\sigma$ and $X_{0}+\sigma$.

For example, in each period, the computer will randomly form groups of three participants and it will assign a random $X_{0}$ to each group. Suppose that a particular group gets an $X_{0}$ equal to 200 points. Therefore, all members of this group will have a CONNECTION VALUE $\left(X_{i}\right)$ from the interval of $200-\sigma$ and $200+\sigma$. That is, the CONNECTION VALUES of agent 1 $\left(X_{1}\right)$, agent $2\left(X_{2}\right)$, and agent $3\left(X_{3}\right)$ will be randomly selected from the interval of 200- $\sigma$ and $200+\sigma$. You will know just your private $X_{i}$. You won't know the value of $X_{0}$ nor will know the CONNECTION VALUES of the other members of your group.

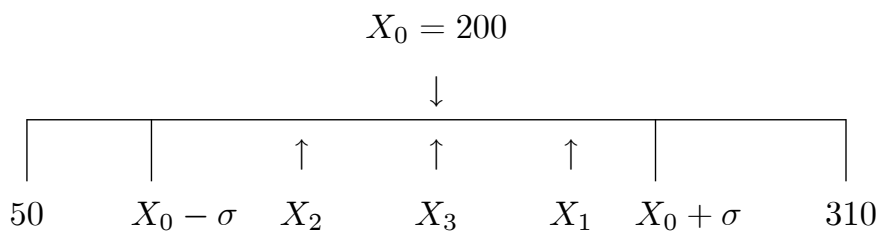

The members of each group should decide whether to connect the other members in their group.

5. Then, you and every member of your group will have to decide whether to request zero, one, or two links. The connection costs and the monetary payoffs that you receive will depend on your decisions to link-request other members of your group and the decisions of the other members of your group to link-request you and among themselves. The costs and the monetary payoffs for each link-request you decide to make will be the sum of the following three payoff components:

5.1 Independent connection payoff: For each member of your group you decide to link-request, you will get the payment of:

\section{ALPHA $\times X_{i}-$ CONNECTION COST}

For each agent you decide to NOT link-request, you will get zero as payoff, even if any other agent has decided to link-request you. 

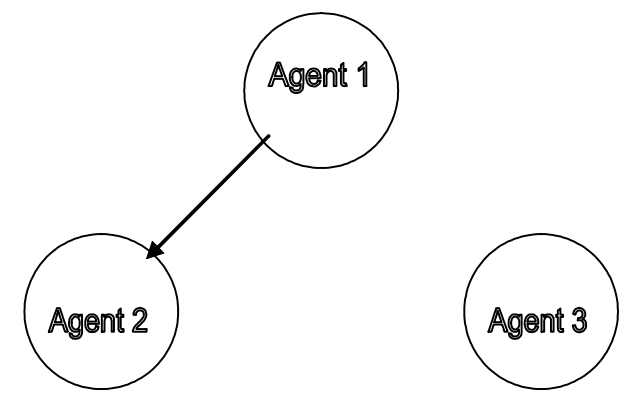

For example, if agent 1 decides to link-request agent 2 and to NOT link-request agent 3, as in the figure above, then agent 1 will get the amount of $A L P H A \times X_{1}-C O N N E C T I O N$ $C O S T$ for link-request agent 2 and zero for NOT link-request agent 3.

5.2. Direct connection payoff: If the agent you have link-request decides to link-request you in return, then you will get the amount of your CONNECTION VALUE $\left(X_{i}\right)$ as an additional payoff.
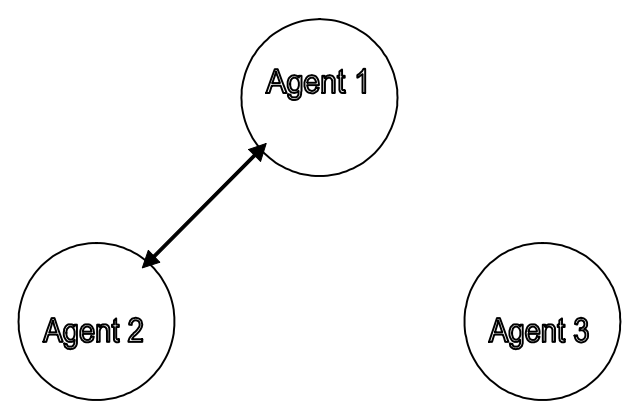

For example, if agents 1 and 2 decide to link-request each other, as in figure above, then agent 1 will get the amount of $A L P H A \times X_{1}-C O N N E C T I O N C O S T$ plus the amount of her $X_{i}$ : That is, $(1+A L P H A) \times X_{1}-C O N N E C T I O N C O S T$. The same will happen with agent 2 .

5.3. Indirect connection payoff: If the agent you have established a direct connection has also established a direct connection with the third agent of your group, you will get the amount of $B E T A \times X_{1}$ as an additional payoff.

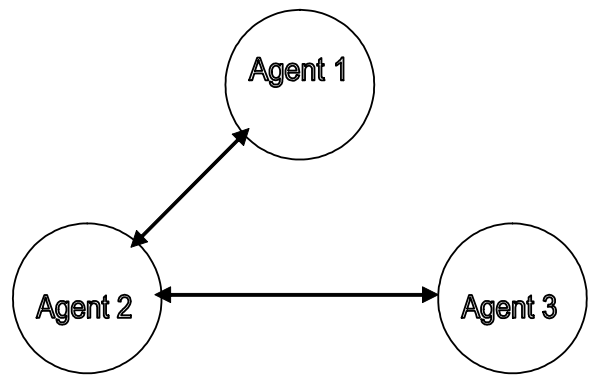


For example, if agents 1 and 2 and agents 2 and 3 decide to link-request each other, as in figure above, then agent 1 will get the sum of all three payoff components: Independent, Direct and Indirect connection payoffs. That is, agent 1 will receive the amount of $A L P H A \times X_{1}-$ CONNECTION COST $+X_{1}+B E T A \times X_{1}$ : That is, $(1+A L P H A+B E T A) \times X_{1}-$ CONNECTION COST

Notice that agent 1 will get her payoff for having an indirect connection with agent 3 through agent 2 even if she has an independent connection with agent 3 . Likewise, the amount an agent can get might be negative or positive depending on her CONNECTION VALUE.

According to the last figure above, agent 1 would get the following payoff.

Payoff Table for Agent 1

\begin{tabular}{|c|c|c|c|c|c|}
\hline Agent & $\begin{array}{l}\text { Independent } \\
\text { Connection }\end{array}$ & $\begin{array}{c}\text { Direct } \\
\text { Connection }\end{array}$ & $\begin{array}{c}\text { Indirect } \\
\text { Connection }\end{array}$ & $\begin{array}{c}\text { Total Payoff } \\
\text { per Connection }\end{array}$ & $\begin{array}{l}\text { Total } \\
\text { Payoff }\end{array}$ \\
\hline Agent 2 & $\begin{array}{c}A L P H A \times X_{1}- \\
C . C O S T\end{array}$ & $X_{1}$ & $B E T A \times X_{1}$ & $\begin{array}{c}A L P H A \times X_{1}+ \\
X_{1}+B E T A \times X_{1}- \\
C . C O S T\end{array}$ & $\begin{array}{c}A L P H A \times X_{1}+ \\
X_{1}+\end{array}$ \\
\hline Agent 3 & 0 & 0 & 0 & 0 & $\begin{array}{c}B E T A \times X_{1}- \\
C . C O S T\end{array}$ \\
\hline
\end{tabular}




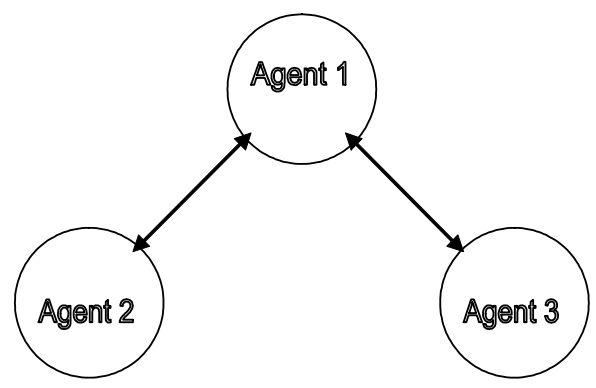

Exercise:

Just for clarification and as an exercise, fill the table indicating how much agent 1 will get given the following graph of connections.

Fill up the following table assuming that the individual CONNECTION VALUE is $X_{1}$.

\begin{tabular}{|c|c|c|c|c|c|}
\hline Agent & $\begin{array}{c}\text { Independent } \\
\text { Connection }\end{array}$ & $\begin{array}{c}\text { Direct } \\
\text { Connection }\end{array}$ & $\begin{array}{c}\text { Indirect } \\
\text { Connection }\end{array}$ & $\begin{array}{c}\text { Total Payoff } \\
\text { per Connection }\end{array}$ & $\begin{array}{c}\text { Total } \\
\text { Payoff }\end{array}$ \\
\hline Agent 2 & & & & & \\
\hline Agent 3 & & & & & \\
\hline
\end{tabular}

6. Initial Capital Balance, Capital Accumulation, and Minimum Capital Balance

Each agent will begin the experiment with a capital balance of 9000 points. Total gains (or loses) in each period will be added (or subtracted) from the previous accumulated capital. If your capital balance in any period is lower than 4500 point, you will not be able to continue participating in the experiment. You will get your capital balance and will have to leave the room.

7. Payment Procedure

Your final payoff will be equivalent to the final capital balance multiplied by 0.33 plus your participation fee. You will receive the amount in check that you can immediately cash at the bank office inside the campus.

¿Are there any questions? 